\title{
One-dimensional high-order compact method for solving Euler's equations
}

\begin{abstract}
In the field of computational fluid dynamics, many numerical algorithms have been developed to simulate inviscid, compressible flows problems. Among those most famous and relevant are based on flux vector splitting and Godunov-type schemes. Previously, this system was developed through computational studies by Mawlood [1]. However the new test cases for compressible flows, the shock tube problems namely the receding flow and shock waves were not investigated before by Mawlood [1]. Thus, the objective of this study is to develop a high-order compact (HOC) finite difference solver for one-dimensional Euler equation. Before developing the solver, a detailed investigation was conducted to assess the performance of the basic third-order compact central discretization schemes. Spatial discretization of the Euler equation is based on flux-vector splitting. From this observation, discretization of the convective flux terms of the Euler equation is based on a hybrid fluxvector splitting, known as the advection upstream splitting method (AUSM) scheme which combines the accuracy of flux-difference splitting and the robustness of flux-vector splitting. The AUSM scheme is based on the third-order compact scheme to the approximate finite difference equation was completely analyzed consequently. In one-dimensional problem for the first order schemes, an explicit method is adopted by using time integration method. In addition to that, development and modification of source code for the one-dimensional flow is validated with four test cases namely, unsteady shock tube, quasi-one-dimensional supersonic-subsonic nozzle flow, receding flow and shock waves in shock tubes. From these results, it was also carried out to ensure that the definition of Riemann problem can be identified. Further analysis had also been done in comparing the characteristic of AUSM scheme against experimental results, obtained from previous works and also comparative analysis with computational results generated by van Leer, KFVS and AUSMPW schemes. Furthermore, there is a remarkable improvement with the extension of the AUSM scheme from first-order to third-order accuracy in terms of shocks, contact discontinuities and rarefaction waves.
\end{abstract}

Keyword: Euler equation; Finite difference methods; Flux vector splitting; Flux-difference splitting; High-order compact schemes 